\title{
Tuberculous Abscess Causing Disruption of the Cranium
}

\author{
G.G. Alvarez, K.B. Sharma, K. Breen, R. Glikstein, H. St. Amand, V. DaSilva
}

Can. J. Neurol. Sci. 2010; 37: 904-905

A 20-year-old male from Nunavut presented to an outpatient clinic complaining of a soft tissue swelling over the left parietal region of his skull. The soft tissue swelling measured approximately $6 \mathrm{~cm} \times 6 \mathrm{~cm}$ and was fluctuant and not warm to touch. Neurological exam was normal. He did not have any discharge from the ear. He did not have a fever, nor did he complain of a headache or stiff neck. He had lost 7 kilograms over the past two months unintentionally. A 22 gauge needle was inserted into the soft tissue swelling and pus was discovered. A skull radiograph demonstrated a radiolucency $2 \mathrm{~cm}$ in diameter without surrounding sclerosis over the left parietal region of the skull (Figure 1). A chest radiograph was done which showed a left hilar opacity extending into the left upper lobe (Figure 2). Tuberculosis (TB) was suspected on the grounds of unintentional weight loss, the chest radiograph abnormalities and the high rates of tuberculosis in the territory. The patient was started on empiric therapy for TB. The patient was unable to produce spontaneous sputum and therefore had sputum induction on three separate days all of which were smear negative for acid fast bacilli. He did not have a cough, night sweats or hemoptysis. Three days later he was referred to a tertiary care center where upon presentation the abscess broke open and drained spontaneously. On presentation his vital signs were normal and he had not developed any new clinical signs or symptoms. A computed tomography scan of the head with contrast was done urgently (Figure 3).

Surgical management was indicated to drain the purulent material, to debride the extra-cranial soft tissue abscess walls, to remove the infected bone edges and to repair the sinus tract communicating the abscess with the exterior of the scalp. The intervention was a combined neurosurgery and plastic surgery operation. A large left temporo-parietal scalp incision allowed us to cut the insertions of the temporal muscle and fascia, and to reflect the soft tissue flap towards the face. Pus was seen escaping the abscess, and samples were obtained for culture. The $1.5 \mathrm{~cm}$ circular bone defect was identified and the bone edge was removed up to the line where the dura was adherent to bone. The walls of the abscess (but not the dura) were debrided, the sinus tract repaired and the wound thoroughly irrigated. A drain was left in the epidural and subtemporal spaces and came out on the second post-operative day.

Cultures from the pus drained from the abscess and from the bone confirmed $M$. tuberculosis which was sensitive to all first line drugs. All spontaneous sputums were smear and culture negative however one of the three induced sputums was culture positive for TB. Computed tomography of the thorax confirmed the left upper lobe consolidation consistent with pulmonary TB. The patient's HIV test was negative.
Despite record low incidence rates of TB in Canada at 4.7 per 100,000 , TB remains a significant problem in Nunavut with an incidence rate of 99.2 per 100,000 in $2007^{1}$. Bone and joint TB make up approximately $3 \%$ of all reported cases in Canada ${ }^{2}$. Initial presentation of tuberculosis involving the skull is very rare as noted by a large retrospective study of 42 cases over a tenyear-period in a tertiary care teaching 1800 bed hospital in

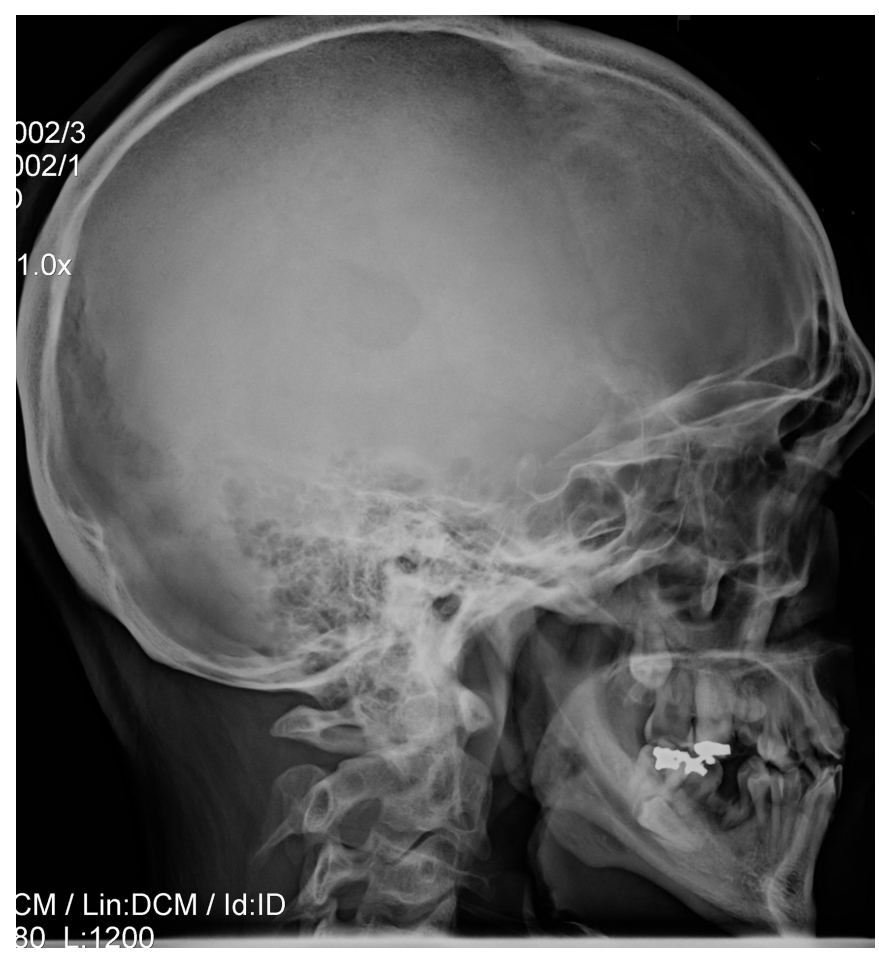

Figure 1: Oval-shaped partially defined hypodensity in the left temporoparietal bone, approximately $2 \mathrm{~cm}$ in diameter. Enlarged left temporoparietal soft tissues.

From the Departments of Medicine, Radiology and Surgery (GGA, KBS, RG, HSA VDS), University of Ottawa at the Ottawa Hospital, Ottawa, Ontario; Department of Medicine (KB), Qikiqtani General Hospital, Iqaluit, Nunavut, Canada.

Received March 30, 2010. Final Revisions Submitted June 9, 2010.

Correspondence to: Krishna B. Sharma, University of Ottawa at The Ottawa Hospital, Division of Respirology, Department of Medicine, 501 Smyth Road, Ottawa, Ontario, K1H 8L6, Canada. 


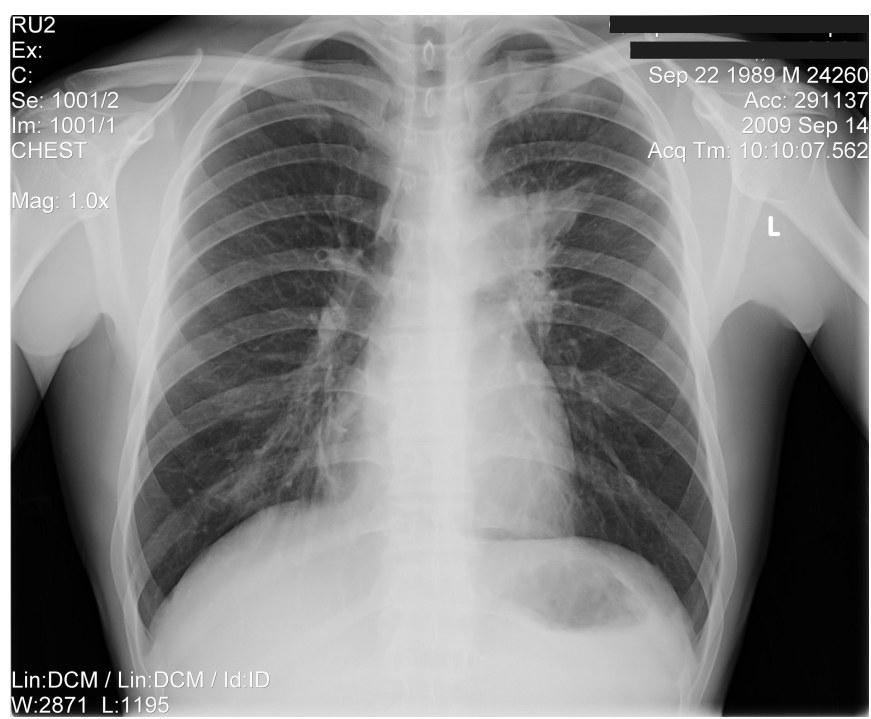

Figure 2: Cavitary pulmonary consolidation in left upper lobe. Pulmonary consolidation in right middle lobe.

India ${ }^{3}$. The case presented is a classic presentation of a rare form of TB which included a painless scalp swelling with minimal erythema and no local warmth, often described as a "cold abscess". In addition, skull TB abscesses commonly affect the parietal bones and occur more commonly in adolescent males ${ }^{3}$.

After primary infection with Mycobacterium tuberculosis, organisms can be 'seeded' through the body via hematogenous spread. These foci of infection are generally contained by local immune response. However, decreased local immunity secondary to HIV infection, renal failure, poor nutrition or immunosuppressive medications may cause reactivation of tuberculosis, sometimes decades in the future. It is believed that most cases of skull TB result from hematogenous or lymphangitic spread from the lungs which is what likely occurred in the case presented. The rarity of this condition is likely explained by the fact that the skull is highly vascular but does not have much in the way of lymphatics ${ }^{4}$. In areas of the world where incidence of tuberculosis is high, bone involvement occurs most frequently in childhood, generally within one year of primary infection; where the incidence of TB is low, it is mainly a disease of adults associated with reactivation ${ }^{2}$. Diagnosis of skeletal tuberculous is often delayed due to the insidious nature of the disease and failure to obtain appropriate specimens for laboratory testing. Differential diagnosis of skeletal TB include bony metastases and non-tuberculous infections such as a fungus like Histoplasma capsulatum or bacteria like Staphylococcus aureus which can cause similar osteomyelitis. As in all other forms of non-respiratory tuberculosis, diagnosis is confirmed with microscopy and culture after needle biopsy or intra-operative sample collection.

The medication regimen for skeletal tuberculosis is the same as that for pulmonary TB, however a longer duration of at least 12 months of therapy is recommended due to concerns about bone penetration ${ }^{2}$. Surgery is indicated in patients with large

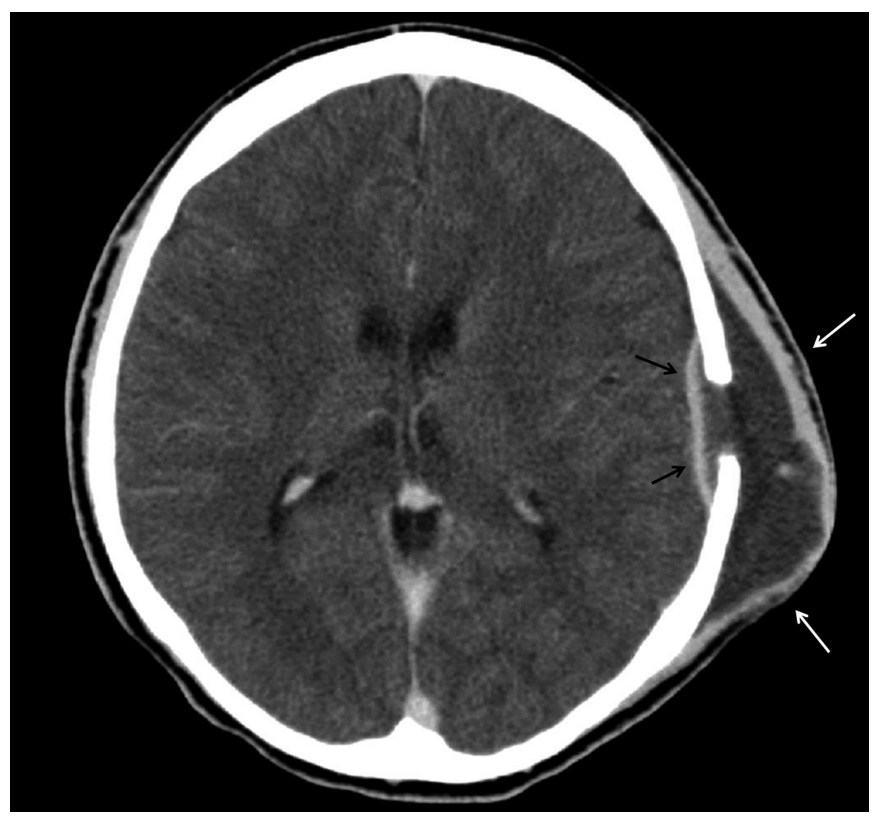

Figure 3: Contrast enhanced axial CT scan of the brain showing a peripherally enhancing large subcutaneous fluid collection (white arrows) communicating with an epidural collection (black arrows) through a non-traumatic bone defect.

extradural collections causing neurological deficits, excision of diseased bone ${ }^{3}$, debridement of co-existing abscesses and posttreatment reconstruction. Ensuring response to treatment involves frequent follow-up to assess the patient's symptoms and monitor for adverse drug reactions or evidence of treatment failure. Radiologic features of bony involvement may initially appear to progress despite appropriate medications, however the treatment regimen should not be changed based on imaging alone $^{5}$. Long-term outcomes of patients with skeletal tuberculosis are good, assuming adherence to the full course of anti-tuberculous medications. The patient tolerated the medications and debridement well and made an excellent recovery with no long-term sequelae.

\section{REFERENCES}

1. phac-aspc.gc.ca [homepage on the Internet]. Public Health Agency of Canada. Tuberculosis in Canada 2007; c2007 [updated 2009 Sept 29; cited 2010 May 29]. Available from: http://www.phacaspc.gc.ca/tbpc-latb/pubs/tbcan07/index-eng.php.

2. phac-aspc.gc.ca [homepage on the Internet]. Public Health Agency of Canada. Canadian Tuberculosis Standards 6th edition; c2007 [updated 2007 Dec 13; cited 2010 May 29]. Available from: http://www.phac-aspc.gc.ca/tbpc-latb/pubs/tbstand07-eng.php.

3. Raut AA, Nagar AM, Muzumdar D, et al. Imaging features of calvarial tuberculosis: a study of 42 cases. Am J Neuroradiol. 2004 Mar;25(3):409-14.

4. Malhotra R, Dinda AK, Bhan S. Tubercular osteitis of skull. Indian Pediatr. 1993 Sep;30(9):1119-23.

5. Boxer DI, Pratt C, Hine AL, McNicol M. Radiological features during and following treatment of spinal tuberculosis. $\mathrm{Br} \mathrm{J}$ Radiol. 1992 Jun;65(774):476-9. 